\title{
BASES LAPAROSCOPICAS DE LA CIRUGIA ROBOTICA.
}

\author{
Octavio A. Castillo ${ }^{1,2}$ y Rafael Sánchez-Salas?.
}

Sección de Endourología y Cirugía Laparoscópica Avanzada'. Clínica Santa María. Santiago. Chile.

Departamento de Urología². Facultad de Medicina de la Universidad de Chile. Santiago. Chile.

\begin{abstract}
Resumen.- La evolución de la terapéutica quirúrgica ha sido vertiginosa y desde los principios clásicos de la cirugía a cielo abierto la evolución ha llevado a través de la laparoscopia a la actual cirugía robótica, en la cual, se ha logrado la aplicación exitosa de los preceptos de ingeniería robótica al quehacer diario del cirujano. El desarrollo de la cirugía mínimamente invasiva, inicialmente liderado por la laparoscopia convencional, constituyó terreno fértil para el desarrollo de técnicas quirúrgicas con el uso del robot. El uso de sistemas automatizados en la cirugía, no es tan novedoso como se pudiese pensar, sin embargo los robots que hoy en día participan en los quirófanos de los centros a nivel mun-
\end{abstract}

dial, constituyen el ejemplo de la tecnología más novedosa y avanzada disponible. La Urología se ha erigido como la especialidad quirúrgica líder en la aplicación de la tecnología para el diagnóstico y resolución de los aspectos clínicos que le competen, y la cirugía robótica no es una excepción. Presentamos nuestra visión de la vigente cirugía automatizada, en el marco de su estrecha relación con la cirugía laparoscopia convencional que la originó.

Palabras clave: Cirugía Robótica. Laparoscopia.

\footnotetext{
Departmento de Urología

Clínica Santa María

Avenida Santa María 0500

7530234 Providencia

Santiago de Chile. (Chile).

octaviocastillo@vtr.net
}

Octavio Castillo

\begin{abstract}
Summary.- The evolution of surgical therapy has been vertiginous, from the classical principles of open surgery to laparoscopy and currently to robotic surgery, in which the principles of robotic engineering have been successfully applied to the surgeon's daily work. The development of minimally invasive surgery, initially led by conventional laparoscopy, was a fertile field for the development of surgical techniques with the robot. The use of automatized systems for surgery is not as new as one could think, but the robots today participating in the main operative rooms worldwide are an example of the newest and most advanced available technology. Urology has become the leading surgical speciality in the application of technologies for diagnosis and treatment of its diseases, and robotic surgery is not an exception. We present our vision about the state of the art in automatized surgery, in the setting of its close relationship with conventional laparoscopic surgery, which originated it.
\end{abstract}

Keywords: Robotic surgery. Laparoscopy. 


\section{INTRODUCCIÓN}

El campo de la urología se ha caracterizado por la innovación en la realización de las diferentes técnicas que implican el tratamiento de los pacientes afectados por patología del tracto genitourinario. Así como el espíritu renovador ha crecido en el campo urológico, de la misma manera, el desarrollo científico y tecnológico de las últimas décadas ha permitido llevar a la realidad las ideas plasmadas dentro del área quirúrgica y en especial en la urología (1).

La definición más completa del término ROBOT, fue realizada por Isaac Asimov: Estructuras mecánicas, en algunos casos similares a los seres humanos, que son capaces de realizar una variedad de tareas, en algunos casos complejas, bajo el comando humano o mediante programación previa (2).

\section{Características de un Robot:}

- Programabilidad. El robot puede almacenar información mediante simbología específica, que permite determinar las tareas que realizará.

- Capacidad Mecánica. El robot puede actuar en su propio medio, y por tanto no sólo almacena y procesa información.

- Flexibilidad. El robot opera usando un rango de programas, manipulando información y datos de diferentes formas.

- Automatismo. El robot puede realizar por si mismo, las funciones para las cuales fue creado.

No es reciente el interés urológico por los mencionados robots. En 1989, Wickham realizó la primera intervención de cirugía robótica urológica con su PROBOT que era capaz de realizar resección endoscópica de la próstata de manera automatizada (3). En 1995, Rovetta y colaboradores (4) realizaron lo que pudo haber sido el primer procedimiento telemanipulado en una experiencia con biopsia prostáti$c a$, y más tarde Kavoussi y Stoianovici presentaron su experiencia con un sistema conocido como TRACKER que permitía realizar punciones renales percútaneas de forma automatizada y de una manera muy efectiva (5).

Actualmente, existen dos tipos de sistemas robóticos: robots endoscópicos y robots telemanipulados. Cada sistema se diferencia en términos de la función que realiza. Los sistemas endoscópicos son útiles en la asistencia para manejo de la cámara durante el procedimiento quirúrgico (EndoAssist, AESOP, LapMan). Los robots telemanipulados o interfa- ses hombre-máquina (Maestro-Esclavo), son los sistemas robóticos completos (Zeus $®$, da Vinci®). Estas interfases están conformadas por la tecnología más avanzada, sin embargo sus elevadas dimensiones y coste han dificultado su amplia difusión (6).

En los momentos actuales la tecnología da Vinci ${ }^{\circledR}$ de Intuitive Surgical, constituye el sistema robótico que ha aunado la suma de los conocimientos y experiencia clínica y tecnológica aportados por sus predecesores AESOP y ZEUS (7).

Al analizar las características de un robot, no es difícil darnos cuenta como la experiencia robótica actual es, sin lugar a dudas la suma de una serie de conocimientos médicos y tecnológicos que se han obtenido a través de siglos, si tomamos en cuenta las descripciones iniciales de automatización presentadas por Leonardo Da Vinci.

Nuestro objetivo en este capítulo, es explicar los cimientos que encuentra la cirugía robótica actual en la laparoscopia convencional, desde el punto de vista de nuestra experiencia personal.

\section{Cirugía Mínimamente Invasiva, lo logrado hasta hoy.}

Después de años, la prueba del tiempo ofreció la razón a la cirugía laparoscópica, que hoy constituye el estándar de tratamiento en patologías quirúrgicas comunes, como lo son la apendicectomía y la colecistectomía. Sin embargo, los inicios no fueron sencillos, y aún hoy están por definirse los estándares quirúrgicos del tratamiento de patologías como el cáncer de próstata, donde aún la cirugía a cielo abierto se mantiene como la primera opción quirúrgica en la gran mayoría de los centros (8).

Los beneficios que aportó la cirugía laparoscópica están hoy claramente definidos (9):

- Pequeñas incisiones, que se traducen en menor dolor y mejor resultado estético.

- Disminución de la hemorragia intraoperatoria, mediante el uso del gas que crea el espacio operatorio.

- Períodos de hospitalización reducidos.

- Magnificación del campo quirúrgico, lo cual ofrece mayor detalle al cirujano.

En el campo urológico los inicios de la cirugía laparoscópica fueron marcados por el uso de la técnica para la realización de la disección linfática a nivel pélvico (10), pero sin duda los avances y de- 
sarrollo quirúrgico que se han realizado en la prostatectomía radical, constituyen lo más representativo de la cirugía laparoscópica urológica. Los últimos 15 años han constituido el período de mayor auge laparoscópico en nuestra especialidad. La realización de los procedimientos laparoscópicos avanzados ha implicado una extensa curva de aprendizaje, debido a los retos técnicos que implica $(11,12)$. El entrenamiento y constancia de los diferentes grupos laparoscópicos a nivel mundial ha permitido que hoy los procedimientos reconstructivos laparoscópicos, casi exclusivos de la urología, se lleven a cabo de forma cotidiana $(13,14)$. Más aún, la laparoscopia se involucrado con éxito en el tratamiento de diversas patologías oncológicas tanto en la urología como en otra especialidades quirúrgicas, permitiendo en nuestros días que los resultados oncológicos laparoscópicos sean mucho más que prometedores y se han constituído como comparables con la cirugía a cielo abierto $(15,16)$.

Los logros y avances han implicado el desarrollo de una serie de reconocidas formas de entrenamiento para la adqusición de las necesarias destrezas que requiere el medio endoscópico:

- Pelvictrainer, para el desarrollo de habilidades básicas.

- Modelos animales y cadavéricos para adquisición de destrezas avanzadas en cada una de las operaciones.

El objetivo final, que en los inicios laparoscópicos constituía superar la pérdida de coordinación ojo-mano, el campo quirúrgico en dos dimensiones, la modificación de la escala visual y la inversión de movimientos, se ha logrado a través de elementos de formación quirúrgica endoscópica que proveen las habilidades para las que fueron creados, son de coste relativamente bajo y simulan en el caso de los modelos animales, las características anatomofisiológicas del ambiente laparoscópico real (17).

Un punto importante a tomar en cuenta es el hecho de que los avances de la cirugía laparoscópi$\mathrm{ca}$, si bien son muy significativos, no representan el grueso de los centros urológicos a nivel mundial y en un interesente estudio presentado por Duchene $y$ colaboradores, se plasma como solo un $38 \%$ de residentes de centros de entrenamiento laparoscópico y cirugía robótica consideran su experiencia laparoscópica como suficiente o satisfactoria (18).

Concomitantemente al desarrollo de la cirugía laparoscópica convencional, se han planteado opciones quirúrgicas que involucran el concepto endoscópico como una alternativa de aplicación de una técnica que sin duda ha requerido inversión significativa de tiempo y recursos para lograr su dominio. Así, conocemos los conceptos de cirugía laparoscópica mano-asistida, aplicados en la urología en el tratamiento del cáncer renal y vesical (19); y el concepto de cirugía asistida por laparoscopia, que se aplica igualmente en el tratamiento del cáncer vesical $(20,21)$.

\section{Cirugía Robótica, la nueva forma de ser mínimamen- te invasivo.}

Si bien la laparoscopia revolucionó el campo de la cirugía, hoy en día, la robótica ha revolucionado el campo de la cirugía mínimamente invasiva. Como hemos mencionado, muchos de los procedimientos laparoscópicos son de alta exigencia técnica y la tecnología ha aportado un medio, mediante el cual, se podría mejorar el desempeño quirúrgico endoscópico; lo que persigue el objetivo de ampliar la utilidad y precisión de la laparoscopia y acortar las curvas de aprendizaje de cada procedimiento (22).

Los aportes de la tecnología robótica al campo laparoscópico son los siguientes:

- Visión de profundidad tridimensional.

- Movimiento multigrado de libertad que imita y mejora la articulación de la mano del cirujano, mediante la utilización del sistema "Endowrist" (Intuitive Surgical, Sunnyvale, CA, USA).

- Posibilidad de tutoría y asistencia a distancia de procedimientos quirúrgicos complejos (23).

El software utilizado por la interfase robótica da Vinci® (Intuitive Surgical, Sunnyvale, CA, USA) permite la simplificación de procedimientos complejos como la anastomosis vesico-uretral en cirugía laparoscópica del cáncer de próstata. De tal manera que los urólogos, se han constituído en los líderes de la cirugía robótica a nivel mundial, y así, el grupo de Detroit liderado por el Dr. Menon ha presentado una importante experiencia con la técnica "Vattikuti Institute Prostatectomy" para el tratamiento del cáncer de próstata localizado, que encuentra sus bases en los principios anatómicos de la prostatectomía radical (24-26).

Otros delicados procedimientos quirúrgicos urológicos, como la pieloplastia, nefrectomías y sacrocolpopexia han sido realizados por técnica robótica con resultados similares a los ofrecidos por la cirugía laparoscópica convencional (27-29). 
Otras especialidades quirúrgicas han verificado los beneficios de la cirugía robótica, como la ginecología oncológica, según lo publicado por Field y colaboradores (30), quienes presentan una serie de 41 pacientes con tumores ginecológicos que fueron tratados con cirugía laparoscópica asistida por robot. Nos llamó la atención que dentro de la descripción de las complicaciones de la serie, se incluyera como tal, el fallo técnico del robot. En este sentido, hacemos la reflexión siguiente:

Si el robot llega a fallar durante el acto quirúrgico, ¿Cuál sería la solución inmediata?. Sin lugar a dudas, las bases que ha sentado la laparoscopia permiten a los cirujanos de hoy, retirar o desacoplar la interfase automatizada y proceder a completar el procedimiento mediante técnicas de laparoscopia convencional. Es claro, que los márgenes de error se irán reduciendo con el tiempo y los posibles fallos técnicos, serán cada vez menores, pero es importante recordar que la parafernalia tecnológica tiene en nuestros días un acceso limitado a los grandes centros de referencia a nivel mundial.

En esta misma idea, es importante mencionar como el llamado docking y undocking (acoplamiento y desacoplamiento) de la interfase robótica constituye un elemento significativo a incluir en el entrenamiento; además de ser tomado en cuenta al momento de evaluar resultados, como ha sido reportado por Vidovszky y colaboradores (31) en una serie de colecistectomía robótica, donde la curva de aprendizaje del procedimiento se relacionó estrechamente con la preparación y/o retiro de la interfase da Vinci®.

La cirugía general además ha implementado el uso del robot, en procedimientos de cirugía bariatrica como ha sido comunicado por Ali y colaboradores (32), quienes han presentado una serie de bypass gástrico en $Y$ de Roux realizado en 30 pacientes. En esta serie, se verificó la importancia de la introducción gradual de la cirugía robótica, con la finalidad de maximizar la eficacia del entrenamiento y disminuir posibles complicaciones. La operación de Nissen para el reflujo gastroesofágico, fue evaluada por Draaisma y colaboradores en un protocolo randomizado, donde se comparó cirugía laparoscópica convencional versus asistida por robot, llegándose a la conclusión de que ambas técnicas son comparables (33).

El futuro (que hoy estamos viviendo) es el resultado de un despliegue tecnológico, que va más allá de lo que muchos imaginamos, pero cuya aplicación debe ajustarse a preceptos quirúrgicos que se han desarrollado a través de siglos de esfuerzo. Nuestra herencia quirúrgica fueron las técnicas pro- badas a cielo abierto, que a través del ímpetu y la constancia, logramos llevar al ambiente quirúrgico endoscópico con resultados adecuados. Para esto, fue de gran ayuda la simbiosis con la tecnología, que sentó las bases de la utilización de instrumentos de alta sofisticación, para el logro de tareas de elevada complejidad. Estamos convencidos de que la cautela con la cual nuestros Profesores miraron la cirugía laparoscópica en sus inicios, estuvo obviamente justificada, y por eso tratamos de enfocar el evento de la cirugía robótica en nuestra especialidad, con igual entusiasmo que precaución, pues a nuestro modo ver, seguiremos haciendo cirugía laparoscópica con la ayuda de una interfase que además de ayudarnos a mejorar nuestro entrenamiento y el de la futuras generaciones, nos otorga la responsabilidad de aclarar cualquier duda en el campo de los resultados oncológicos y de las complicaciones que puedan aún mantenerse en el campo urológico.

Es probable que la ventajas robóticas, de la visión tridimensional y la extrema maniobrabilidad, nos permita de nuevo revisar los objetivos específicos de cada una de la intervenciones y como consecuencia, realizar un mayor esfuerzo en lograr tales tareas, mientras al mismo tiempo nos abocamos a evitar cualquier posible morbilidad asociada al acto quirúrgico.

Es importante mantener en mente que las ventajas operatorias ofrecidas mediante la aplicación del robot mantienen al cirujano como beneficiario exclusivo. Los ayudantes de la cirugía, no sólo deberán estar preparados para su actuación en el ambiente laparoscópico convencional, sino que además deben, mantener una clara familiaridad con el robot, con el cual compartirán el espacio quirúrgico. Entrenar residentes en el uso de los sistemas robóticas presenta nuevos retos a los responsables de la formación de venideras generaciones de cirujanos de todas las especialidades. En este sentido, un abordaje sistemático en la formación de los cirujanos con las mencionadas técnicas, debe permitir un entrenamiento seguro y efectivo (34).

La difícil sencillez que observamos en los videos presentados en las series robóticas, sin duda alguna constituye, el elemento a cultivar en los próximos años; de tal manera que los beneficios se traduzcan en el bienestar de nuestro fin último, el paciente.

\section{CONCLUSIONES}

La cirugía robótica ofrece a la cirugía la tecnología más novedosa para su aplicación en la resolución de exigentes procedimientos quirúrgicos. 
Los beneficios de la cirugía minimamente invasiva, como son: las pequeñas incisiones, disminución de la hemorragia y menor período de convalecencia, han sido probadas por la laparoscopia convencional. La cirugía robótica hereda estos beneficios, y aporta un mejor campo visual quirúrgico con mayor y mejor maniobrabilidad lo que debe permitir ofrecer mejores resultados en las series clínicas en la medida del tiempo.

\section{AGRADECIMIENTOS}

A Doña Margarita Barrueto Naranjo y a Don Mauricio Mejías por su valiosa colaboración editorial.

\section{BIBLIOGRAFÍA Y LECTURAS RECOMENDADAS (*lectura de interés $y$ ** lectura fundamental)}

*1. HOZNEK, A.; KATZ, R.; GETTMAN, M. y cols.: "Laparoscopic and robotic surgical training in Urology." Curr. Urol. Rep., 4: 130, 2003.

2. CLARKE, R.: "Asimov's laws for robotics: implications for information technology". IEEE Computer, January, pp 313-320. Part 1-2 December, pp 53-61, 1993.

3. DAVIES, B.L.; HIBBERD, R.D.; COPTEOAT, M.J. y cols.: "A surgeon robot prostatectomy- a laboratory evaluation". J. Med. Eng. Technol., 13: 273, 1989.

4. ROVETTA, A.; SALA, R.; COSMI, F. y cols.: "Telerobotic surgery in a transatlantic experiment: application in laparoscopy". Proceedings of telemanipulator technology and space telerobotics, Boston, 1993.

5. CHALLACOMBE, B.J.; KAVOUSSI, L.R.; DASGUPTA, P.: "Transoceanic telerobotic surgery". BJU Int., 92: 678, 2003.

6. LONG, J.A.; DESCOTES, J.L.; SKOWRON, O. y cols.: "Use of robotics in laparoscopic urological surgery: state of the art". Prog. Urol., 16: 3, 2006.

**7. CHALLACOMBE, B.J.; SHAMIM KHAN, M.; MURPHY, D. y cols.: "The history of robotics in urology”. World J. Urol., 24: 120, 2006.

8. GETTMAN, M.T.; BLUTE, M.L.: "Critical comparison of laparoscopic, robotic and open radical prostatectomy: techniques, outcomes and cost". Curr. Urol. Rep., 7: 193, 2006.

9. RIGDON, J.L.: "Robotic-assisted laparoscopic radical prostatectomy". AORN J., 84: 760, 2006.
10. SCHUESSLER, W.W.; VANCAILLIE, T.G.; REICH, H. y cols: "Transperitoneal endosurgical lymphadenectomy in patients with localized prostate cancer". J. Urol., 145: 988, 1991.

*11. VILLEGAS, L.; SCHNEIDER, B.E.; CALLERY, M.P. y cols.: "Laparoscopic skills training". Surg. Endosc., 17: 1879, 2003.

12. FREDE, T.; HAMMADY, A.; KLEIN, J. y cols.: "The radius surgical system - A new device for complex minimally invasive procedures in Urology?". Eur. Urol., 2006.

13. CHUNG, S.Y.; MELDRUM, K.; DOCIMO S.G.: "Laparoscopic assisted reconstructive surgery: a 7 year experience.” J. Urol., 171: 372, 2004.

14. KAOUK, J.H.; GILL, I.S.: "Laparoscopic reconstructive urology". J. Urol., 170: 1070, 2003.

15. BROWN, J.A.; LEWIS, R.W.: "A multi-institution, minimally invasive urological oncology fellowship: a critical assessment of the clinical training and academic benefits". J. Urol., 176: 2619, 2006.

16. COLOMBO, J.R.; HABER, G.P.; RUBINSTEIN, M. y cols.: "Laparoscopic surgery in urological oncology: brief overview". Int. Braz. J. Urol., 32: 504, 2006.

*17. VAN VELTHOVEN, R.F.; HOFFMANN, P.: "Methods for laparoscopic training using animal". Current Urol. Reports, 7: 114, 2006.

18. DUCHENE, D.A.; MOINZADEH, A.; GILL, I.S. y cols.: "Survey of residency training in laparoscopic and robotic surgery". J. Urol., 176: 2158, 2006.

19. PETERSON, A.C.; LANCE, R.S.; AHUJA, S.K.: "Laparoscopic hand assisted radical cystectomy with ileal conduit urinary diversion". J. Urol., 168: 2103, 2002.

20. CATHELINEAU, X.; ARROYO, C.; ROZET, F. y cols.: "Laparoscopic assisted radical cystectomy: the Montsouris experience after 84 cases". Eur. Urol., 47: 780, 2005.

21. BASILLOTE, J.B.; ABDELSHEHID, C.; AHLERING, T.E. y cols.: "Laparoscopic assisted radical cystectomy with ileal neobladder: a comparison with the open approach". J. Urol., 172: 489, 2004.

**22. AHLERING, T.E.; SKARECKY, D.; LEE, D. y cols.: "Successful transfer of open surgical skills to a laparoscopic environment using a robotic interface: initial experience with laparoscopic radical prostatectomy". J. Urol., 170: 1738, 2003.

23. SEBAJANG, H.; TRUDEAU, P.; DOUGALL, A. y cols.: "The role of telementoring assistance in the provision of laparoscopic colorectal surgery in rural areas". Surg. Endosc., 20: 1389, 2006.

*24. HERNAL, A.K.; MENON, M.: "Robotics in Urology”. Cur. Op. Urol., 14: 89, 2004. 
**25. MENON, M.; TEWARI, A.; PEABODY, J. y cols: "Institute Prostatectomy technique. The VIP learn”. J. Urol., 169: 2289, 2003.

26. VILLAVICENCIO MAVRICH, H.: "Da Vinci advanced robotic laparoscopic surgery: origin and current clinical application in urology, and comparison with open and laparoscopic surgery". Actas Urol. Esp., 30: 1, 2006.

27. WEISE, E.S.; WINFIELD, H.N.: "Robotic computer-assisted pyeloplasty versus conventional laparoscopic pyeloplasty". J. Endourol., 20: 813, 2006.

28. LEE, R.S.; BORER, J.G.: "Robotic surgery for ureteropelvic junction obstruction”. Curr. Opin. Urol., 16: 291, 2006.

29. MENON, K.: "Robotics in laparoscopic urology: Minim Invasive Ther Allied". Technol., 14: 62, 2005.

30. FIELD, J.B.; BENOIT, M.F.; DINH, T.A. y cols.: "Computer-enhanced robotic surgery in gynecologic oncology". Surg. Endosc., 19, 2006.

31. VIDOVSZKY, T.J.; SMITH, W.; GHOSH, J. y cols.: "Robotic cholecystectomy: learning curve, advantages, and limitations". J. Surg. Res, 136: 172, 2006.

*32. ALI, M.R.; RASMUSSEN, J.; BHASKERRRAO, B.: "Teaching robotic surgery: a stepwise approach". Surg. Endosc., 20, 2006.

33. DRAAISMA, W.A.; RUURDA, J.P.; SCHEFFER, R.C. y cols.: "Ramdomized clinical trial of standard laparoscopic vs robot-assisted laparoscopic Nissen fundoplication for gastro-oesopahgeal reflux disease". Br. J. Surg., 93: 1351, 2006.

34. RASHID, H.H.; LEUNG, Y.Y.; RASHID, M.J. y cols.: "Robotic surgical education: a systematic approach to training urology residents to perform robotic-assisted laparoscopic radical prostatectomy". Urology, 68: 75, 2006. 